\title{
Traumatic aortic root rupture leading to acute aortic insufficiency and acute Type A aortic dissection.
}

\author{
Subash Nepal${ }^{1}$, Vijay Raj $^{1}$, Debanik Chaudhuri $^{2}$, and Stephany Barreto ${ }^{1}$ \\ ${ }^{1}$ SUNY Upstate Medical University \\ ${ }^{2}$ State University of New York Upstate Medical University - University Hospital
}

January 12, 2021

\begin{abstract}
A 17-year-old male was admitted for the management of multiple fractures after sustaining blunt thoracic trauma. He was hemodynamically stable and without any cardiac symptoms. He was admitted with fracture of T4 end plate, manubrium and left first rib, right pulmonary contusion, left apical pneumothorax and pneumomediastinum. The patient underwent echocardiography and cardiac CT angiogram for the work up of aortic injury as the patient had new aortic regurgitation murmur, troponin rise and RBBB. He was found to have aortic root rupture, type A aortic dissection and acute severe aortic insufficiency. The patient underwent surgical aortic valve and root replacement with Bentall procedure with good outcome.
\end{abstract}

\section{INTRODUCTION}

Motor vehicle accidents are the most common cause of blunt chest trauma (1). A study showed that $34 \%$ of the fatalities resulting from blunt chest trauma were from thoracic aortic injury and $80 \%$ at the crash scene had mortality from exsanguination into the mediastinum (2). While myocardial contusion leading to troponin leak is the most common injury and may lead to impaired ventricular systolic function, valve injury is rare (3).

\section{CASE REPORT}

A 17-year old male presented to an outside hospital with blunt trauma to the chest during a dirt bike accident. He had multiple injuries including T4 endplate compression fracture, right pulmonary contusion, left apical pneumothorax, pneumomediastinum, fracture of manubrium and left first rib. The patient denied any chest pain, palpitations or shortness of breath. He was hemodynamically stable and not hypoxemic. Physical examination revealed bounding peripheral pulses with a heart rate of 105, wide pulse pressure with blood pressure of 122/44 and tachypnea with respiratory rate of 26. Cardiac auscultation revealed normal heart sounds and early diastolic murmur in the aortic area. The patient was initially admitted to the trauma service for the management of the fractures.

\section{Differential diagnosis}

* Traumatic deceleration injury with aortic root rupture

* Traumatic aortic regurgitation

\section{Investigations}

Laboratory analysis revealed elevated troponin $\mathrm{T}$ of $3.3 \mathrm{ng} / \mathrm{mL}$ and $\mathrm{EKG}$ revealed new right bundle branch block. CT angiography of the thorax revealed prominent aortic sinus (image 1), pneumomediastinum, an aortic root disruption with Stanford type A dissection with hemopericardium (image 2) and rupture of the 
epicardium of the right ventricle and adventitia of the pulmonary artery. The epicardial hematoma was contained but the patient had significant intrapericardial bleeding and clot. TTE revealed dissection flap in the ascending aorta extending into the right sinus of Valsalva (image 6 and media 1 ) and severe aortic regurgitation with holodiastolic flow reversal in the abdominal aorta (image 3, image 4 and image 5, media 2). Intraoperative TEE revealed dilated aortic root to $4 \mathrm{~cm}$, dissection flap in the ascending aorta extending into the right sinus of Valsalva and severe aortic regurgitation (image 7, media 3).

\section{Management}

The patient was tachycardic and was started on low dose esmolol drip. Midline sternotomy was performed which revealed that the heart and mediastinum were encased in clot and bulging pericardium which was removed with pericardiotomy. Direct visual examination revealed an intact aortic arch with rupture at the sino-tubular ridge extending to the left coronary ostium. The entire posterior aspect of the ascending aorta was transected with complete separation of the adventitia, media and intima with dissecting aneurysm and contained periaortic hematoma formation. Left coronary ostium was repaired, $23 \mathrm{~mm}$ valved St. Jude conduit was placed, ascending aorta was repaired and both coronary arteries were re-implanted. Intraoperative TEE post-Bentall procedure revealed a competent aortic valve and good flow in the coronary arteries. The patient was discharged home with uneventful postoperative stay.

\section{DISCUSSION}

The aortic root injury is a rare but fatal complication of blunt thoracic injury if not repaired early. The valvular injury is uncommon and the aortic valve injury is the most common valvular injury, while the mitral and tricuspid valves are rarely involved (4). It may lead to type A aortic dissection and aortic valve disruption, either due to commissure detachment or rupture of the cusps leading to acute aortic regurgitation (4). Injuries to the aorta can be classified into four types: intimal tear only, large intimal flap with intramural hematoma, pseudoaneurysm and traumatic aortic rupture (TAI) (5). Blunt trauma to the chest causes injury predominantly to the parts of the heart and blood vessel that are tethered. The sites of normal anatomic tethering in aorta are aortic isthmus, which is the segment of proximal descending aorta at the confluence of mobile arch and fixed descending aorta and the aortic root, which is the confluence between the mobile heart and fixed ascending aorta (5). The differential deceleration forces that are created in these anatomic areas due to blunt chest trauma lead to shearing of tissues at the junction and make them prone to injury (5). A normal high aortic root pressure gradient across closed aortic valve during ventricular diastole further contributes to traumatic aortic leaflet injury (6).Aortic isthmus is the most common site of TAI accounting for about $80 \%$ of the cases reported (7). Prognosis is better with isthmus rupture $(19.8 \%$ survival) versus ascending aortic rupture(4\% survival), due to complex anatomy at the root and survival depending solely on contained hematoma at that location (8). Thoracic aortic injury has high mortality and a study has shown that $80 \%$ of victims died at the scene, underscoring the importance of urgent surgery (2). Our patient was hemodynamically stable when presented to our center as he had contained periaortic hematoma and did well after surgery.

CT angiography of the thorax and TEE are the primary diagnostic tools (9). TEE is beneficial as it helps in bedside evaluation of the extent of valve injuries, integrity of sino-tubular junction, pericardial and myocardial injuries (10). It also helps in immediate intraoperative evaluation of successful valve implantation, good coronary flow and the integrity of the repaired structures.

Open surgical repair aided by bypass-perfusion technique has been the preferred modality of repair for the ascending aortic injury. A Bentall procedure which comprises using a valve conduit to replace the ruptured aortic valve, aortic root and ascending aorta is the gold standard with good outcome (11).

\section{Follow up}

The patient is doing well after discharge. He denies any chest pain, palpitation, shortness of breath, syncope or effort intolerance.

\section{CONCLUSION}


In conclusion, aortic root injury should be suspected in a patient with blunt chest trauma. The auscultatory finding of aortic regurgitation murmur along with radiographic evidence of intrathoracic injury should be investigated by echocardiography followed by $\mathrm{CT}$ of the thorax if clinically indicated. Urgent surgery for acute aortic root rupture leading to aortic dissection and severe aortic insufficiency is lifesaving and has a good outcome.

\section{REFERENCES}

1. Liman ST, Kuzucu A, Tastepe AI, Ulasan GN, Topcu S. Chest injury due to blunt trauma. European journal of cardio-thoracic surgery : official journal of the European Association for Cardio-thoracic Surgery 2003;23:374-8.

2. Teixeira PG, Inaba K, Barmparas G et al. Blunt thoracic aortic injuries: an autopsy study. The Journal of trauma 2011;70:197-202.

3. TENZER ML. The Spectrum of Myocardial Contusion: A Review. Journal of Trauma and Acute Care Surgery 1985;25:620-627.

4. Kan CD, Yang YJ. Traumatic aortic and mitral valve injury following blunt chest injury with a variable clinical course. Heart 2005;91:568-570.

5. Azizzadeh A, Keyhani K, Miller CC, 3rd, Coogan SM, Safi HJ, Estrera AL. Blunt traumatic aortic injury: initial experience with endovascular repair. Journal of vascular surgery 2009;49:1403-8.

6. Obadia JF, Tatou E, David M. Aortic valve regurgitation caused by blunt chest injury. Br Heart J 1995;74:545-547.

7. Parmley LF, Mattingly TW, Manion WC, Jahnke EJ, Jr. Nonpenetrating traumatic injury of the aorta. Circulation 1958;17:1086-101.

8. PARMLEY LF, MATTINGLY TW, MANION WC, JAHNKE EJ. Nonpenetrating Traumatic Injury of the Aorta. Circulation 1958;17:1086-1101.

9. Hiratzka LF, Bakris GL, Beckman JA et al. 2010 ACCF/AHA/AATS/ACR/ASA/SCA/SCAI/SIR/STS/SVM guidelines for the diagnosis and management of patients with Thoracic Aortic Disease: a report of the American College of Cardiology Foundation/American Heart Association Task Force on Practice Guidelines, American Association for Thoracic Surgery, American College of Radiology, American Stroke Association, Society of Cardiovascular Anesthesiologists, Society for Cardiovascular Angiography and Interventions, Society of Interventional Radiology, Society of Thoracic Surgeons, and Society for Vascular Medicine. Circulation 2010;121:e266-369.

10. Sun X, Hong J, Lowery R et al. Ascending aortic injuries following blunt trauma. Journal of cardiac surgery 2013;28:749-55.

11. German DS, Shapiro MJ, Willman VL. Acute aortic valvular incompetence following blunt thoracic deceleration injury: case report. The Journal of trauma 1990;30:1411-2. 


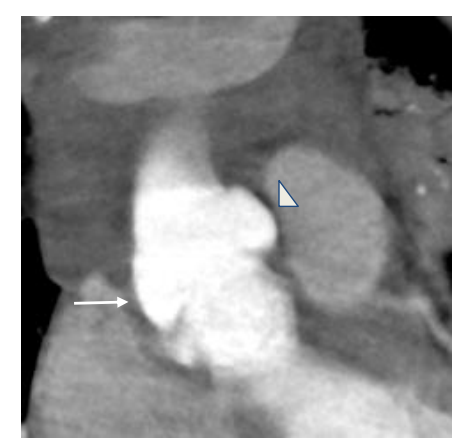




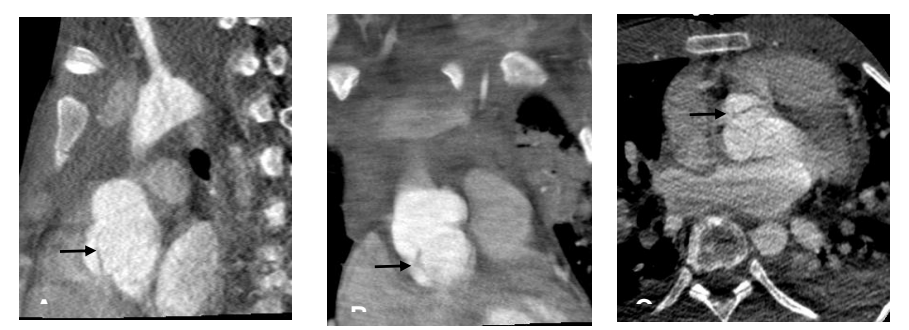




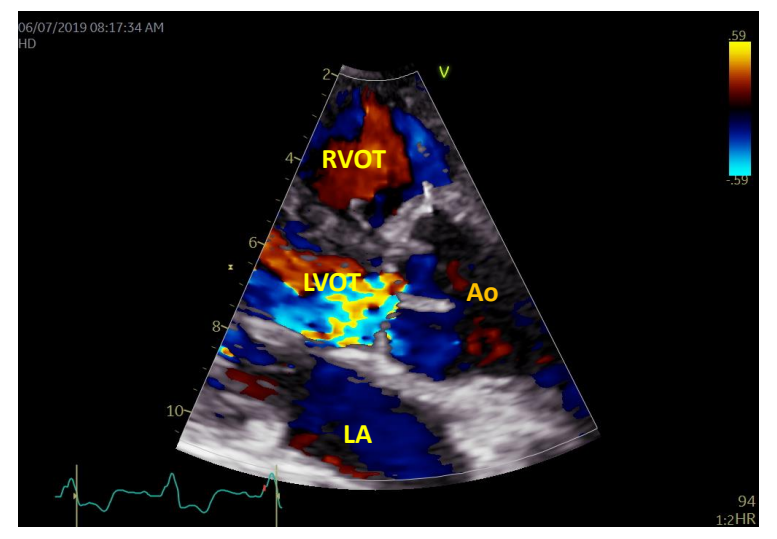




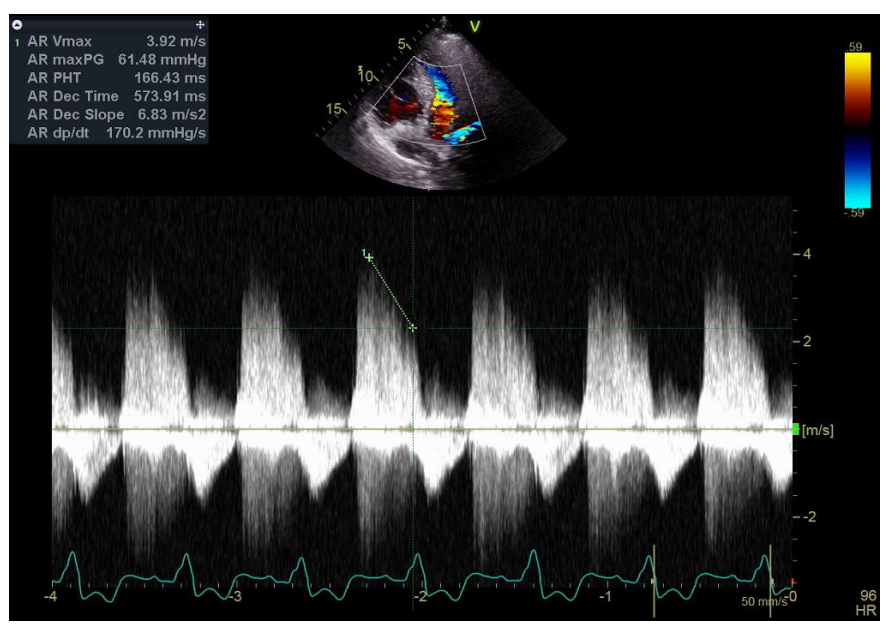




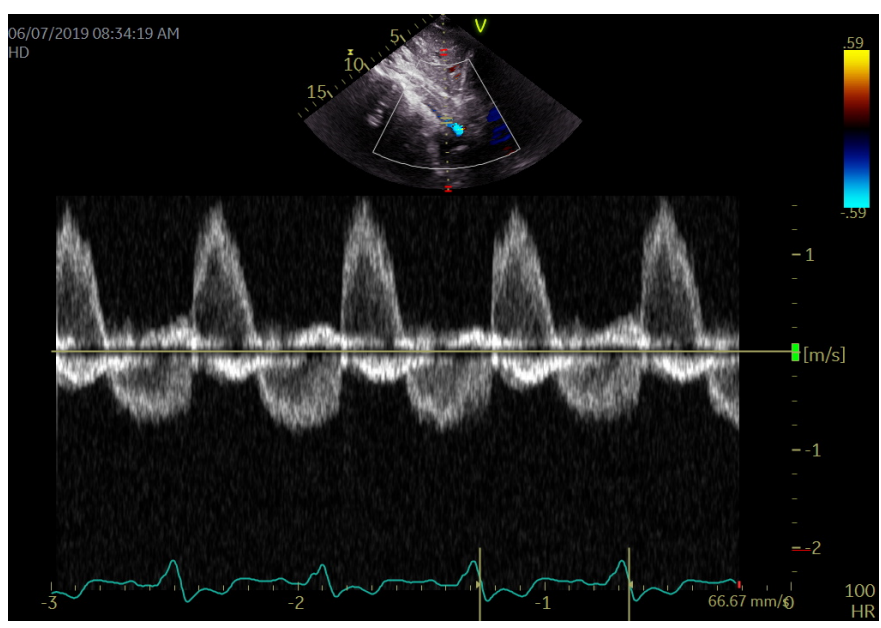



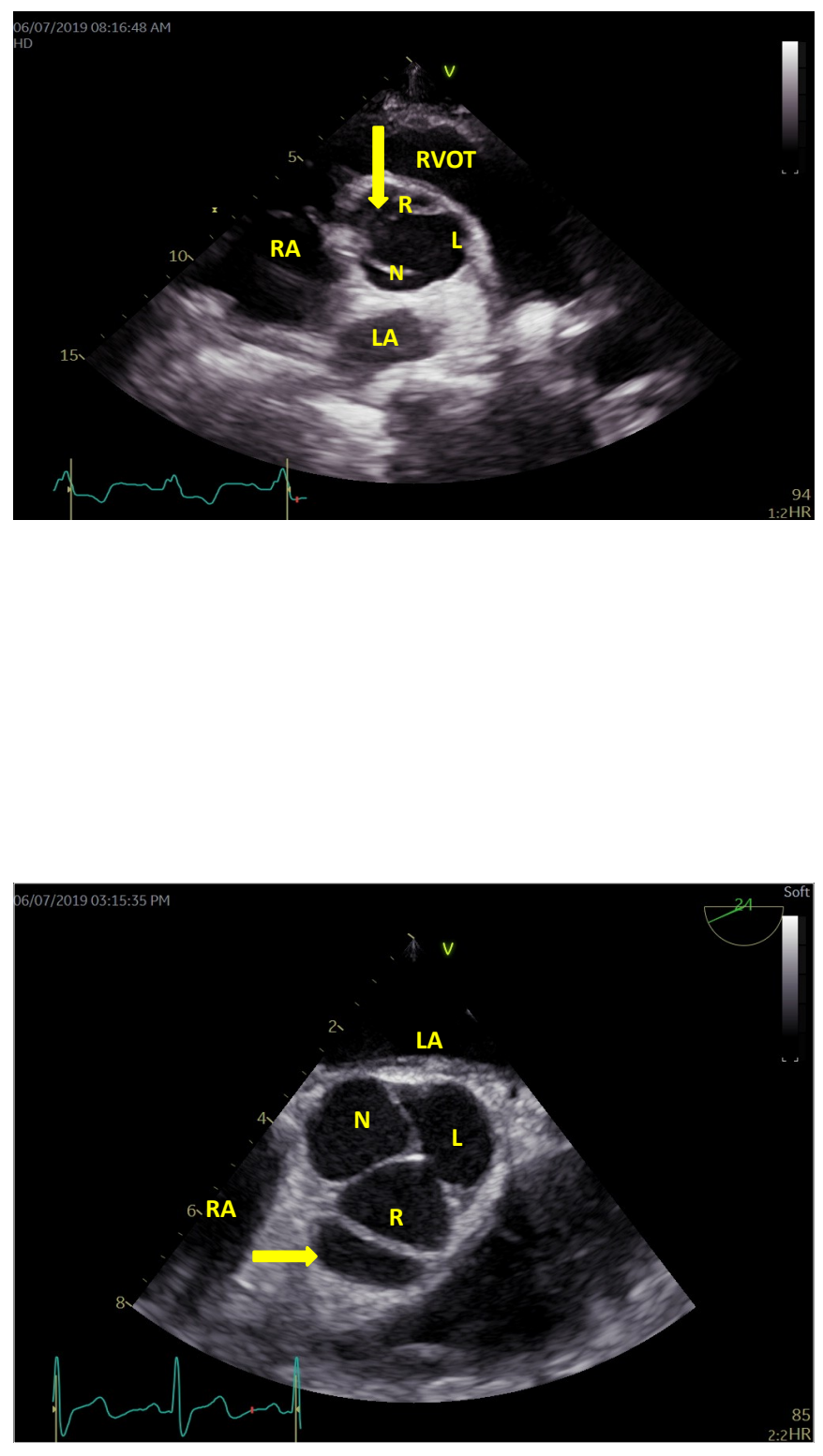